\title{
Inactivated influenza vaccine is safe for children and adults with stable asthma
}

American Lung Association Asthma Clinical Research Centers. The safety of inactivated influenza vaccine in adults and children with asthma. N Engl J Med 2001 Nov 22;345:1529-36.

\section{QUESTION: In children and adults with stable asthma, is the inactivated trivalent split virus influenza vaccine safe?}

\section{Design}

Randomised (allocation concealed), blinded (clinicians and patients), placebo controlled crossover trial with follow up to 14 days after each injection.

\section{Setting}

19 centres in the US.

\section{Patients}

2032 patients who were 3-64 years of age, had stable asthma, and had been taking prescribed treatment for asthma within the preceding 12 months. Exclusion criteria included hypersensitivity to egg products or thimerosal, inability to use the peak flow meter properly, lack of a telephone, history of Guillain-Barré syndrome, influenza vaccine in the previous 6 months, and a temperature $\geqslant 38.0^{\circ} \mathrm{C}$ within 24 hours before enrolment. 1952 patients (96\%) (mean age $30 \mathrm{y}, 62 \%$ girls and women) received vaccine and placebo injections and completed both 14 day postinjection diaries.

\section{Intervention}

Patients were allocated in a crossover design to receive an injection of inactivated (heat killed trivalent split virus) influenza type $\mathrm{A}$ and $\mathrm{B}$ vaccine and an injection of placebo 4 weeks apart.

\section{Main outcome measures}

The primary outcome measure was an exacerbation of asthma (defined as $\geqslant 1$ of the following: $\geqslant 30 \%$ decrease in peak expiratory flow rate [PEFR] from the second highest morning PEFR [personal best] measured during the study, new or increased use of systemic corticosteroids, unscheduled healthcare visits for asthma symptoms, and increased use of rescue medication) within 14 days after an injection. Secondary outcomes included a decrease of $\geqslant 20 \%$ in PEFR from the personal best rate during the 14 days after each injection, average morning PEFR, symptoms thought to be associated with the vaccine or placebo injection (rhinitis, sore throat, cough, headache, body aches, fever, body chills, or fatigue), number of days without symptoms of asthma, amount of time lost from work or school because of illness, and an increase in the dose of medication used for long term control.

\section{Main results}

Analysis was by intention to treat. Exacerbations of asthma within 14 days after vaccine or placebo injections did not differ (table). Exacerbation rates were also similar in groups of patients defined according to age and severity of asthma. More patients reported body aches after the vaccine than after the placebo injection $(25 \%$ v 21\%, $\mathrm{p}<0.001)$. The vaccine and placebo injections did not differ for other secondary outcomes.

\section{Conclusion}

In children and adults with stable asthma, inactivated influenza vaccine was as safe as placebo.

\section{COMMENTARY}

Of an estimated 26 million people in the US diagnosed with asthma, 10.6 million have had an asthma attack in the past year. ${ }^{1}$ Several studies have shown that respiratory viruses including para influenza, influenza, adenovirus, and coronavirus are associated with increased asthma symptoms. ${ }^{2}$

Influenza vaccine is the principal method of preventing and controlling influenza, and its use is recommended for patients with asthma in many national vaccination programmes. However, the benefits and safety of administering the influenza vaccine in patients with asthma have been questioned. ${ }^{4}$

The American Lung Association Asthma Clinical Research Centers used rigorous methods in this study to address the question of safety of the inactivated influenza vaccine in a tertiary academic clinic population of patients with asthma. They included a large sample with a wide age range and varying degrees of asthma severity. The crossover design allowed a mean of 22 days between injections.

The frequency of asthma exacerbations was similar in the 2 groups, even when patients were divided into subgroups according to demographic characteristics, asthma severity, smoking status, and lung function. Even though this study was done in a tertiary care setting, which often results in recruitment of only patients with severe illness, many patients had mild to moderate asthma; hence, study findings may be applied to patients with milder asthma.

This study has shown that using the inactive trivalent influenza vaccine is safe in patients with varying severity of asthma. The safety of other influenza vaccine types in asthma is yet to be proved.

\author{
B Haleema Shakur, RGN, MSc \\ Howard M Branley, MBChB, MSc \\ Department of Respiratory Medicine (NHLI Division), \\ Imperial College School of Medicine, Hammersmith Campus, London, UK
}

1 Mannino DM, Homa DM, Akinbami LJ, et al. Surveillance for asthma-United States, 1980-1999. Mor Mortal Wkly Rep CDC Surveill Summ 2002;51(SS01):1-13.

2 Tuffaha A, Gern JE, Lemanske RF Jr. The role of respiratory viruses in acute and chronic asthma. Clin Chest Med 2000;21:289-300.

3 Cates CJ, Jefferson TO, Bara AI, et al. Vaccines for preventing influenza in people with asthma. Cochrane Database Syst Rev 2000;(4):CD000364.

4 Nicholson KG, Nguyen-Van-Tam JS, Ahmed AH, et al. Randomised placebo-controlled crossover trial on effect of inactivated influenza vaccine on pulmonary function in asthma. Lancet 1998;351:326-31. 